\title{
NORMAL ANALYTIC FUNCTIONS AND A QUESTION OF M. L. CARTWRIGHT
}

\section{DOUGLAS M. CAMPBELL AND GEORGE PIRANIAN}

In an address before the London Mathematical Society, M. L. Cartwright asked whether there exists a normal analytic function in $|z|<1$ with an infinite radial limit at $z=1$ but with a derivative that has no radial limit at $z=1$. W. K. Hayman and D. A. Storvick [4] answered this in the affirmative, using a geometric construction to exhibit a univalent function with the required property. In this paper, we give three different explicit examples relevant to Cartwright's question.

\section{A logarithmic function with a Blaschke disturbance}

Let $\left\{z_{n}\right\} \quad\left(0<z_{1}<z_{2}<\ldots<1\right)$ be an interpolating sequence, and let $B(z)=\prod_{n=1}^{\infty}\left(\frac{z_{n}-z}{1-z_{n} z}\right)$ be the Blaschke product with simple zeros at the points of $\left\{z_{n}\right\}$. Then $B$ is a real-valued function on the segment $[0,1)$ of the real line, and it changes sign at each point $z_{n}$. Thus $\left\{B^{\prime}\left(z_{n}\right)\right\}$ is a sequence of real numbers, negative when $n$ is odd, positive when $n$ is even. Since $\left\{z_{n}\right\}$ is an interpolating sequence, there exists a number $\delta>0$ such that $\left(1-\left|z_{n}\right|^{2}\right)\left|B^{\prime}\left(z_{n}\right)\right| \geqslant \delta \quad(n=1,2, \ldots)\left[3 ;\right.$ p. 148]. Thus $B^{\prime}\left(z_{2 n}\right) \geqslant$ $\delta /\left(1-z_{2 n}^{2}\right)$ and $B^{\prime}\left(z_{2 n+1}\right) \leqslant-\delta /\left(1-z_{2 n+1}^{2}\right)$. Consider the normal (Bloch) function

$$
f(z)=\frac{\delta}{2} \log \frac{1+z}{1-z}-B(z)
$$

Clearly, $\lim _{x \rightarrow 1} f(x)=\infty$. On the other hand, $f^{\prime}\left(z_{2 n}\right) \leqslant 0$ and $f^{\prime}\left(z_{2 n+1}\right) \geqslant 2 \delta /\left(1-z_{2 n+1}^{2}\right)$. By continuity, $f^{\prime}$ vanishes between $z_{2 n}$ and $z_{2 n+1}$, while $f^{\prime}\left(z_{2 n+1}\right) \rightarrow \infty$. Thus $f^{\prime}$ has no finite or infinite radial limit at $z=1$.

\section{An example obtained by integration}

Our second example illustrates a connection between the non-Euclidean distance of consecutive points of an exponential interpolating sequence $\left\{z_{n}\right\}$ and the behaviour of the Blaschke product that vanishes at each point $z_{n}$. Let $\left\{z_{n}\right\}$ be an exponential interpolating sequence on the positive real line [3;p. 156]. For simplicity let us suppose that $z_{n}=1-c^{n}(0<c<1)$. Let $B(z)$ be the Blaschke product with a simple zero at each $z_{n}$. Since the pseudo-non-Euclidean distance from $z_{n}$ to $z_{n+1}$ satisfies the condition

$$
\frac{z_{n+1}-z_{n}}{1-z_{n} z_{n+1}}=\frac{1-c}{1+c-c^{n+1}}>\frac{1-c}{1+c}
$$

Received 26 March, 1979.

The second author gratefully acknowledges support from the National Science Foundation.

[J. LONDON MATh. SOC. (2), 20 (1979), 467-471] 
we can find points $z_{n}^{\prime}$ and $z_{n+1}^{\prime \prime}$ such that $z_{n}<z_{n}^{\prime}<z_{n+1}^{\prime \prime}<z_{n+1}$,

$$
\frac{z_{n}^{\prime}-z_{n}}{1-z_{n}^{\prime} z_{n}}=\frac{1}{4} \frac{1-c}{1+c}=\frac{z_{n+1}-z_{n+1}^{\prime \prime}}{1-z_{n+1} z_{n+1}^{\prime \prime}},
$$

and the non-Euclidean distance $\rho\left(z_{n}^{\prime}, z_{n+1}^{\prime \prime}\right)$ from $z_{n}^{\prime}$ to $z_{n+1}^{\prime \prime}$ is bounded below by a positive number $d$ depending on $c$ but not on $n$. Since $\left\{z_{n}\right\}$ is an interpolating sequence, there exists a number $\delta>0$ such that

$$
\prod_{\substack{j=1 \\ j \neq k}}^{\infty}\left|\frac{z_{j}-z_{k}}{1-z_{j} z_{k}}\right| \geqslant \delta \quad(k=1,2, \ldots)
$$

[3; p. 148]. Thus, for $z_{n}^{\prime}<z<z_{n+1}^{\prime \prime}$,

$$
\begin{aligned}
B^{2}(z) & =\prod_{j=1}^{\infty}\left(\frac{z_{j}-z}{1-z_{j} z}\right)^{2} \\
& \geqslant\left(\frac{z_{n}-z}{1-z_{n} z}\right)^{2}\left(\frac{z_{n+1}-z}{1-z_{n+1} z}\right)^{2} \prod_{j=1}^{n-1}\left(\frac{z_{j}-z_{n}}{1-z_{j} z_{n}}\right)^{2} \prod_{j=n+2}^{\infty}\left(\frac{z_{j}-z_{n+1}}{1-z_{n+1} z_{j}}\right)^{2} \\
& \geqslant\left(\frac{1}{4} \frac{1-c}{1+c}\right)^{2}\left(\frac{1}{4} \frac{1-c}{1+c}\right)^{2}\left(\prod_{\substack{j=1 \\
j \neq s}}^{\infty} \mid \frac{z_{j}-z_{n}}{1-z_{j} z_{n}}\right)^{2}\left(\prod_{\substack{j=1 \\
n \neq n+1}}^{\infty}\left|\frac{z_{j}-z_{n+1}}{1-z_{j} z_{n+1}}\right|\right)^{2} \\
& \geqslant \delta^{4}(1-c)^{4} / 256(1+c)^{4} \\
& \equiv A>0 .
\end{aligned}
$$

Consider the normal (Bloch) function

$$
F(z)=\int_{0}^{z} B^{2}(w)\left(1-w^{2}\right)^{-1} d w
$$

Clearly, $F^{\prime}\left(z_{n}\right)=0$ for each $n$. On the other hand

$$
\begin{aligned}
F\left(z_{n+1}\right) & \geqslant \sum_{j=1}^{n} \int_{z^{\prime} j}^{z^{\prime \prime} j_{j+1}} B^{2}(w)\left(1-w^{2}\right)^{-1} d w \\
& \geqslant \sum_{j=1}^{n} A \int_{z^{\prime} j}^{z^{\prime \prime} j_{j+1}}\left(1-w^{2}\right)^{-1} d w \\
& \geqslant \sum_{j=1}^{n} A \cdot d \\
& =n A d,
\end{aligned}
$$


and therefore $\lim F(x)=\infty$. Since $F(x) \rightarrow \infty$ as $x \rightarrow 1$, there exists a sequence of points $x_{n}$ on $[0,1)$ such that $\lim _{n \rightarrow \infty} F^{\prime}\left(x_{n}\right)=\infty$. Thus $\lim _{x \rightarrow 1} F^{\prime}(x)$ fails to exist.

\section{A univalent example}

Our third example is inspired by the domain described by Hayman and Storvick; but instead of analyzing a univalent mapping defined by a precisely preassigned domain, we use a univalent function described by a simple formula.

Let $\left\{\theta_{n}\right\}$ denote a decreasing sequence of positive numbers such that $\theta_{1}<1$ and $\sum\left|\log \theta_{n}\right|^{-1 / 2}<\infty$. For $n=1,2, \ldots$, let $g_{n}(z)=e^{-i \theta_{n}} \log \frac{1}{1-z e^{i \theta_{n}}}+e^{i \theta_{n}} \log \frac{1}{1-z e^{-i \theta_{n}}}$, where the two logarithmic expressions represent principal values. Obviously the function

$$
g(z)=z+\sum_{n=1}^{\infty} g_{n}(z) /\left|\log \theta_{n}\right|
$$

is holomorphic in the unit disc $D$.

In the formula

$$
g^{\prime}(z)=1+\sum_{n=1}^{\infty}\left(1 /\left(1-z e^{i \theta_{n}}\right)+1 /\left(1-z e^{-i \theta_{n}}\right)\right) /\left|\log \theta_{n}\right|
$$

the real part of each term under the summation sign is positive; therefore $g$ is a univalent, close-to convex Bloch function in $D$.

It is easy to see that the domain $g(D)$ consists roughly of $D$ together with pairs of narrow fingers reaching to infinity in the directions $e^{i \theta_{n}}$ and $e^{-i \theta_{n}}$. The positivity of $g^{\prime}$ on the segment $[0,1)$ guarantees that $\lim _{r \rightarrow 1} g(r)$ exists. Since $g(r) \geqslant r+\sum_{n=1}^{m} g_{n}(r) /\left|\log \theta_{n}\right|$, we have the inequality

$$
\lim _{r \rightarrow 1} g(r) \geqslant \lim _{m \rightarrow \infty}\left(1+\sum_{n=1}^{m} g_{n}(1) /\left|\log \theta_{n}\right|\right)
$$

which together with $g_{n}(1) \sim 2\left|\log \theta_{n}\right|$ proves that $\lim g(r)=\infty$. Since $g(r) \rightarrow \infty$ as $z \rightarrow 1$, the derivative $g^{\prime}(r)$ is not bounded on $[0,1)$.

We subject the $\theta_{n}$ to an additional requirement. Assuming that $\theta_{1}, \theta_{2}, \ldots, \theta_{j}$ have been chosen, let $D_{j}$ denote the set of points in $D$ where $\left|g_{j}^{\prime}(z)\right| \geqslant\left|\log \theta_{j}\right|^{1 / 2}$. Since $\left|g^{\prime}{ }_{j}(z)\right|<\left|z-e^{i \theta_{j} \mid}\right|^{-1}+\left|z-e^{-i \theta_{j}}\right|^{-1}$, the set $D_{j}$ lies in the union of the two overlapping discs $\left|z-e^{ \pm i \theta_{j}}\right| \leqslant 2\left|\log \theta_{j}\right|^{-1 / 2}$. However $g_{j}^{\prime}(1)=1$ which implies there is an open disc around $z=1$ which is disjoint from $D_{j}$. We choose $\theta_{j+1}$ so that $D_{j+1}$ lies in this open 
disc and is therefore disjoint from $D_{j}$. Each neighborhood of $z=1$ contains a point $z_{m}$ of $[0,1)-\bigcup_{n=1}^{\infty} D_{n}$ satisfyir $; g_{n}^{\prime}\left|g_{n}^{\prime}\left(z_{m}\right)\right|<\left|\log \theta_{n}\right|^{1 / 2}$ for every $m=1,2, \ldots$. Therefore,

$$
\begin{aligned}
\left|g^{\prime}\left(z_{m}\right)\right| & \leqslant 1+\sum_{n=1}^{\infty}\left|g_{n}^{\prime}\left(z_{m}\right)\right| / / \log \theta_{n} \mid \\
& <1+\sum_{n=1}\left|\log \theta_{n}\right|^{1 / 2} .
\end{aligned}
$$

This inequality together with $g^{\prime}$ being unbounded on $[0,1)$ proves that $g^{\prime}$ has no radial limit at $z=1$ and completes the proof.

The function constructed by Hayman and Storvick in [4] has infinite planar area and maps the disc onto a Jordan domain (on the Riemann sphere). A slight modification of our example produces a function which has finite planar area and which also maps the disc onto a Jordan domain (on the Riemann sphere). It suffices to move the logarithmic branch points slightly beyond the unit circle. To be precise let

$$
G(z)=z+\sum_{n=1}^{\infty}\left\{e^{-i \theta_{n}} \log \frac{1}{1+\theta_{n}^{2}-z e^{i \theta_{n}}}+e^{i \theta_{n}} \log \frac{1}{1+\theta_{n}^{2}-z e^{-i \theta_{n}}}\right\} /\left|\log \theta_{n}\right| .
$$

Then $G(z) \rightarrow \infty$ as $z \rightarrow 1$. If $\left\{\theta_{n}\right\} \rightarrow 0$ fast enough, then the sequence $\left\{G^{\prime}\left(1-\theta_{n}^{2}\right)\right\}$ is bounded, the stereographic image of $G(D)$ is a Jordan domain, and the domain $G(D)$ has finite planar area.

\section{Concluding remarks}

We note that the existence of a normal analytic function in $D$ for which $\lim f(z)$ is finite while $\lim _{x \rightarrow 1} f^{\prime}(x)$ fails to exist is easily established. The bounded function $(1-z)^{1+i}$ tends to zero as $z \rightarrow 1$ radially while its derivative (which is also bounded) has no radial limit at 1 . In fact, there exists a univalent function $f$ and a set $E$ of measure $2 \pi$ such that for each $\theta$ in $E$ the radial limit $f\left(r e^{i \theta}\right)$ exists while $\lim _{r \rightarrow 1} f^{\prime}\left(r e^{i \theta}\right)$ fails to exist. Simply let

$$
f(z)=\int_{0}^{2} \exp \left(\frac{1}{8} \sum_{n=1}^{\infty} w^{2^{n}}\right) d w .
$$

Since $\left(1-|z|^{2}\right)\left|f^{\prime \prime}(z) / f^{\prime}(z)\right|<1 / 2$, the function $f(z)$ is univalent $[6 ; p .172]$ and therefore has a radial limit almost everywhere $[2 ;$ p. 56]. On the other hand, $\log f^{\prime}(z)=\frac{1}{8} \sum_{n=1}^{\infty} z^{2 n}$ has no finite radial limits by the high-indices theorem of Hardy and Littlewood (see [1] for an elegant proof). Thus $f^{\prime}(z)$ can have only 0 and $\infty$ as radial 
limits. Since $f^{\prime}(z)$ is normal, each radial limit is an angular limit. Thus $f^{\prime}$ can have only 0 and $\infty$ as angular limits. It follows from Privalov's uniqueness theorem for angular limits $[2 ;$ p. 146] that the radial limits 0 and $\infty$ can occur only on a set of measure 0 . Therefore there exists a set $E$ of measure $2 \pi$ such that $\lim _{r \rightarrow 1} f\left(r e^{i \theta}\right)$ exists for all $\theta$ in $E$ while $\lim _{r \rightarrow 1} f^{\prime}\left(r e^{i \theta}\right)$ exists for no $\theta$ in $E$.

\section{References}

1. K. G. Binmore, "Analytic functions with Hadamard gaps", Bull. London Math. Soc., 1 (1969), 211-217.

2. E. F. Collingwood and A. J. Lohwater, The theory of cluster sets (Cambridge University Press, Cambridge, 1966).

3. P. L. Duren, Theory of $H^{\tilde{p}}$ spaces, (Academic Press, New York, 1970).

4. W. K. Hayman and D. A. Storvick, "A question of M. L. Cartwright", J. London Math. Soc. (2), 5 (1972), 419-422.

5. O. Lehto and K. I. Virtanen, "Boundary behaviour and normal meromorphic functions", Acta Math., 125 (1970), 269-298.

6. Ch. Pommerenke, Univalent functions (Vandenhoeck and Ruprecht, Göttengen, 1975).

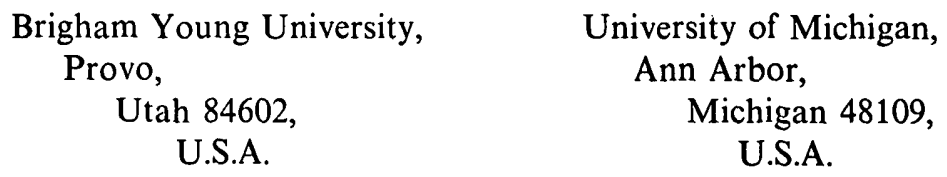

